\title{
Recent patent applications in single-molecule technologies
}

\author{
Julien Muzard
}

O

ver the last 10 years, single-molecule research and nano-biosensor tools have emerged as a new class of analytical and ultrasensitive technology for the design of nextgeneration methods capable of identifying and discriminating between individual molecules. A panel of inventions in this field recently disclosed to patent offices worldwide by biotechnology companies and public research agencies is detailed herein (Table 1).
The ambitious goal of sequencing individual human genomes for about $\$ 1,000$ apiece is likely to be achieved within the next few years. One of the best candidates is the nanopore platform, whose ability to detect and manipulate chemical and molecular entities (such as nucleic acids) at a very-high-resolution, or 'single-molecule,', level is one of the bridges linking together chemistry, nanomaterial science, cellular and molecular biology and biophysics. This promises to have a considerable impact on biotechnology, with clear applications for the real world.

\section{ACKNOWLEDGMENTS}

The author acknowledges the Bionanosciences group and HEA, PRTLI5, Nanoremedies program for financial support and research infrastructure.

\section{COMPETING FINANCIAL INTERESTS}

The author declares no competing financial interests.

Table 1 Recent patent applications in single-molecule technologies

\begin{tabular}{|c|c|c|c|c|c|}
\hline Patent number & Description & Assignee & Inventor & $\begin{array}{l}\text { Priority } \\
\text { application date }\end{array}$ & Publication date \\
\hline US 2011249259 & $\begin{array}{l}\text { Methods and apparatus for single-molecule sensing and } \\
\text { molecular analysis of analytes (nucleic acids, proteins, } \\
\text { polypeptides, peptides, lipids and polysaccharides) } \\
\text { by optical spectroscopy in solid-state nanopores in a } \\
\text { transmission-based approach. }\end{array}$ & $\begin{array}{l}\text { Louvain Catholic } \\
\text { University (Louvain, } \\
\text { Belgium), IMEC } \\
\text { (Louvain, Belgium) }\end{array}$ & $\begin{array}{l}\text { Van Dorpe P, } \\
\text { De Vlaminck I, } \\
\text { Lagae L, Borghs G }\end{array}$ & $12 / 9 / 2008$ & 10/13/2011 \\
\hline US 2011212437 & $\begin{array}{l}\text { Single-molecule sequencing with two distinct chemistry } \\
\text { steps. }\end{array}$ & $\begin{array}{l}\text { Pacific Biosciences } \\
\text { (Menlo Park, CA, } \\
\text { USA) }\end{array}$ & $\begin{array}{l}\text { Emig R, Jia L, } \\
\text { Hanes J, Sebo L }\end{array}$ & 2/18/2010 & 9/1/2011 \\
\hline $\begin{array}{l}\text { US } 2011200989 \text {, } \\
\text { WO } 2011091043\end{array}$ & $\begin{array}{l}\text { Single-molecule nucleic acid sequencing using } \\
\text { multiphoton fluorescence excitation. }\end{array}$ & $\begin{array}{l}\text { Life Technologies } \\
\text { (Carlsbad, CA, USA) }\end{array}$ & $\begin{array}{l}\text { Janaway GA, } \\
\text { Inman CE, } \\
\text { Beechem J }\end{array}$ & $1 / 19 / 2010$ & $7 / 28 / 2011$ \\
\hline US 20110177496 & $\begin{array}{l}\text { Compositions, methods and apparatus for DNA } \\
\text { sequencing in a two-electrode chamber by field-switch } \\
\text { sequencing. }\end{array}$ & $\begin{array}{l}\text { Pacific Biosciences } \\
\text { (Menlo Park, CA, } \\
\text { USA) }\end{array}$ & $\begin{array}{l}\text { Williams JGK, } \\
\text { Anderson JP }\end{array}$ & 4/30/2004 & $7 / 21 / 2011$ \\
\hline $\begin{array}{l}\text { US } 2009029477 \\
\text { US } 7972858\end{array}$ & $\begin{array}{l}\text { Methods for analyzing polymer molecules and } \\
\text { high-throughput readout of DNA and RNA molecules } \\
\text { with single-molecule sensitivity. }\end{array}$ & $\begin{array}{l}\text { Harvard College } \\
\text { (Cambridge, MA, } \\
\text { USA) }\end{array}$ & $\begin{array}{l}\text { Mathe J, Meller A, } \\
\text { Eid JS }\end{array}$ & $8 / 13 / 2004$ & $7 / 5 / 2011$ \\
\hline US 20110160078 & $\begin{array}{l}\text { Digital counting of individual molecules by stochastic } \\
\text { attachment of diverse labels. }\end{array}$ & $\begin{array}{l}\text { Affymetrix (Santa } \\
\text { Clara, CA, USA) }\end{array}$ & Fodor S, Fu GK & $12 / 15 / 2009$ & $6 / 30 / 2011$ \\
\hline WO 2011047680 & $\begin{array}{l}\text { Immunochemical detection, visualization and } \\
\text { quantification of single unit of biological or chemical } \\
\text { targets. }\end{array}$ & $\begin{array}{l}\text { Dako (Glostrup, } \\
\text { Denmark) }\end{array}$ & Lohse J & 10/20/2009 & 4/28/2011 \\
\hline $\begin{array}{l}\text { US } 2009159812 \\
\text { US } 7914734\end{array}$ & $\begin{array}{l}\text { Electromagnetic radiation-based scanning analyzer } \\
\text { for single-molecule detection and methods of use for } \\
\text { diagnostics with zero carryover between samples. }\end{array}$ & $\begin{array}{l}\text { Singulex (Alameda, } \\
\text { CA, USA) }\end{array}$ & Livingston RA & 12/19/2007 & $3 / 29 / 2011$ \\
\hline US 2011053286 & $\begin{array}{l}\text { An apparatus and improved method for detecting and } \\
\text { monitoring (bio)-chemical reactions or interactions at } \\
\text { the single-molecule level. }\end{array}$ & $\begin{array}{l}\text { Life Technologies } \\
\text { (Carlsbad, CA, USA) }\end{array}$ & $\begin{array}{l}\text { Battulga } \mathrm{N}, \\
\text { Reddy } \mathrm{M}, \\
\text { Hardin } \mathrm{SH}\end{array}$ & $7 / 20 / 2006$ & $3 / 3 / 2011$ \\
\hline US 2011021383 & $\begin{array}{l}\text { Apparatuses for real-time RNA or DNA single-molecule } \\
\text { sequence determination. }\end{array}$ & $\begin{array}{l}\text { Life Technologies } \\
\text { (Carlsbad, CA, USA) }\end{array}$ & $\begin{array}{l}\text { Hardin S, } \\
\text { Briggs J, Tu S-C, } \\
\text { Gao X, Willson R }\end{array}$ & $7 / 7 / 2000$ & 1/27/2011 \\
\hline WO 2009065635 & $\begin{array}{l}\text { Single cell-based reporter assay to monitor gene } \\
\text { expression patterns with a rapid and high spatiotempo- } \\
\text { ral resolution. }\end{array}$ & $\begin{array}{l}\text { Pasteur Institut } \\
\text { (Paris) }\end{array}$ & $\begin{array}{l}\text { Mhlanga M, } \\
\text { Enninga J, } \\
\text { Sansonetti P, } \\
\text { Nehrbass U }\end{array}$ & $9 / 12 / 2007$ & $5 / 28 / 2009$ \\
\hline
\end{tabular}

Source: US Patent and Trademark Office, Espacenet, JP and EP patent offices. The status of each application is slightly different from country to country. 